\title{
Computed Tomography (CT) Findings in Imminent Cardiac Failure
}

\author{
Puneet Garg ${ }^{1}$, Mahesh Kumar Mittal ${ }^{2}$, Nisha Rathi ${ }^{3}$, Shabnam Bhandari Grover ${ }^{4}$ \\ ${ }^{1}$ Assistant Professor, ${ }^{2}$ Professor, ${ }^{3}$ Junior Resident, ${ }^{4}$ Professor and HOD, Department of Radiodiagnosis, VMMC \& Safdarjung \\ Hospital, New Delhi, India
}

Corresponding author: Dr Puneet Garg, 40C MIG, DDA flats, Motia Khan, Paharganj, New Delhi-110055

DOI: 10.21276/ijcmsr.2018.3.2.22

How to cite this article: Puneet Garg, Mahesh Kumar Mittal, Nisha Rathi, Shabnam Bhandari Grover. Computed tomography (CT) findings in imminent cardiac failure. International Journal of Contemporary Medicine Surgery and Radiology. 2018;3(2):B89-B91.

\section{A B S T R A C T}

Introduction: Imaging a patient who is in imminent cardiac arrest, is not of common occurrence but cases can be seen in busy emergency departments, where contrast enhanced computed tomography (CT) examinations are being carried routinely. These CT examinations are conducted to assess the internal injuries especially in patients of poly-trauma who are vulnerable to undergo hypovolemic shock. Findings of layering of contrast in right sided heart chambers, inferior vena cava (IVC), hepatic and renal veins with parenchymal blush due to contrast reflux are salient imaging findings with associated poor contrast filling of aorta and systemic arteries.

Case report: In this case report we describe one such situation where a young, male patient who suffered multiple injuries developed cardiac arrest during the CT examination and was successfully revived while the patient was still in CT room. He showed similar findings of imminent cardiac arrest as described above.

Conclusion: Specific imaging findings of contrast layering in the venous circulation with parenchymal reflux and reduced systemic arterial contrast filling are helpful in diagnosing a rare condition of imminent cardiac failure during CT scanning and adequate resuscitative measures can be undertaken while the patient is still in CT room.

Key words: Heart Arrest; Tomography; Vena Cava; Hepatic Veins; Renal Veins

\section{INTRODUCTION}

Imaging of polytrauma patients by computed tomography is becoming a routine practice in busy emergency departments. Although imaging should be done in stable patients but due to dependency on cross sectional imaging for rapid diagnosis and triage of the patients, many patients undergo CT examination. Imminent cardiac failure is a condition where the heart is on the verge of complete failure and is not able to push the blood into systemic circulation. Certain specific imaging findings have been described in the literature like layering of the contrast in IVC, hepatic veins, renal veins and contrast reflux into the parenchyma. These findings should be recognized at the earliest by the radiologist or the technician so that prompt measures can be undertaken to save the life of the patient who is in imminent cardiac failure during CT examination.

\section{CASE REPORT}

We present a case of 25-year-old male patient who suffered multiple injuries in a road traffic accident who was brought to the emergency department in unconscious state. After initial assessment and treatment, he was found to have multiple bilateral rib fractures with subcutaneous emphysema. On further examination he was found to have sternal fracture, right clavicular fracture, right femoral shaft fracture. On chest $\mathrm{x}$-ray he had bilateral lung consolidation however no pneumothorax could be seen. Patient was put on intravenous crystalloids and blood transfusion to maintain the blood pressure whichwas consistently below 90 / 60 mmHg. He was taken up for emergency CT angiography of chest and abdomen to rule out any internal injury and hemorrhage which could result in hypovolemic shock.

While undergoing computed tomographic examination, the patient collapsed with a rapid fall in blood pressure with systolic pressures of $60 \mathrm{mmHg}$ and unrecordable diastolic pressure. Soon the patient went into asystole and carotid pulse was absent. The arterial blood gas analysis showed $\mathrm{pH}$ of $6.99, \mathrm{PO} 2$ of 31.5 and $\mathrm{PCO} 2$ of 66.5 . Cardiopulmonary resuscitation measures were taken and patient was revived with cardiac activity on electrocardiogram and blood pressure of $84 / 60 \mathrm{~mm}$ of $\mathrm{Hg}$. Patient regained sinus rhythm with however with tachycardia. Infusion of non-adrenaline and dopamine was started in view of cardiogenic shock.

Evaluation of the CT scan revealed multiple and characteristic findings in the form of dependent pooling of contrast in the inferior vena cava (figure 1), contrast reflux into hepatic veins with hepatic parenchymal blush (figure $2)$, contrast reflux into right renal vein with veno-interstitial reflux (figure 2 and 3 ). The contrast within the left side of the heart chambers was adequately seen and opacification of aorta was also achieved however the caliber of the aorta was significantly reduced likely due to poor inflow of blood 

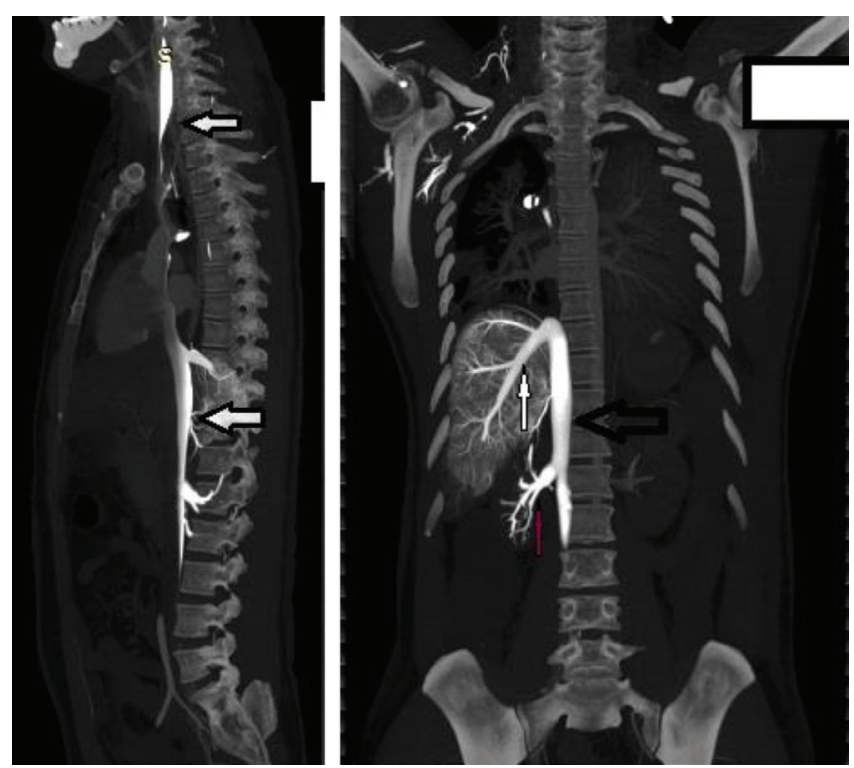

Figure-1: Saggital maximum intensity projection (MIP), thick slab shows dependant contrast pooling into superior and inferior vena cava (white arrows). Figure-2: Coronal maximum intensity projection (MIP) thick slab shows reflux of contrast into IVC (black arrow), into hepatic veins (white arrow) with hepatic parenchymal blush and also into right renal vein (pink arrow)

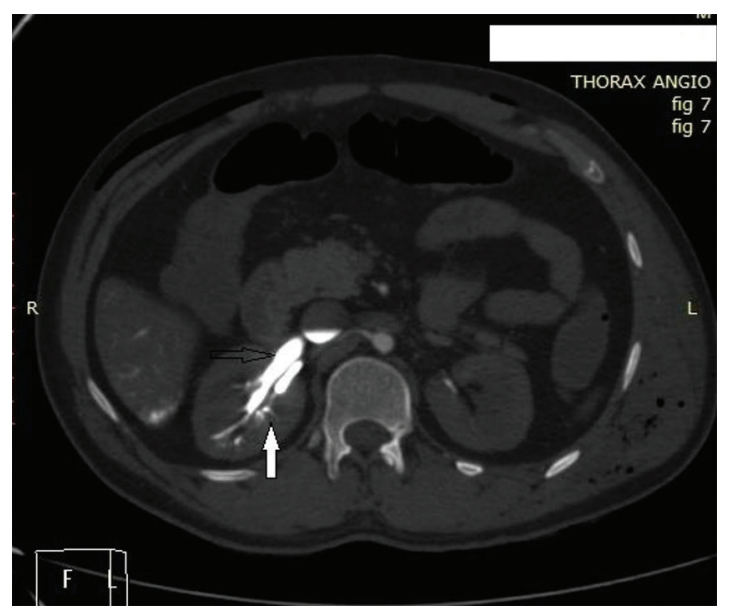

Figure-3: Axial contrast angiography image through the level of kidneys reveal dense contrast opacification of right renal vein (black horizontal arrow) and venointerstitial reflux (vertical white arrow). Dependant contrast level is also seen within the IVC.

(figure 4). Right-sided heart chambers were comparatively enlarged than the left side. Associated other findings of rib fractures, sternal fracture, right clavicular fracture, subcutaneous emphysema and bilateral lung consolidation were also noted (figure 4). There was no evidence of any pneumothorax or pneumomediastinum and the abdomen did not reveal any significant abnormality. So the diagnosis of imminent cardiac failure was made on imaging.

The patient was shifted to intensive care unit where he suffered multiple episodes of cardiac arrest with successful resuscitation. The patient was on positive inotropic support while in the intensive care unit in view of cardiogenic shock, however patient succumbed to his injuries after 2 days.

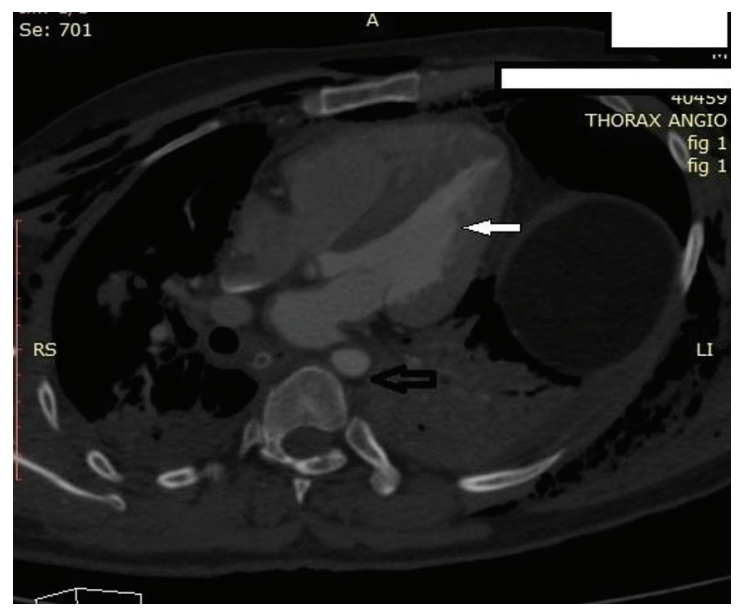

Figure-4: Axial image through the chest shows adequate contrast within the left ventricle (white arrow) and descending thoracic aorta however with reduced caliber (black arrow). Associated findings of surgical emphysema and lung consolidation can be seen.

\section{DISCUSSION}

Computed tomography (CT) is widely used as the imaging modality to scan patients in both in-patients and out- patient department. Similarly nowadays CT has a pivotal role to triage patients in emergency department. In the setting of trauma services, CT is helpful in rule out any grevious internal injury which could lead to demise of the patient. In patients of poly-trauma, whole body CT scan including head, neck, chest and abdomen with pelvis often with CT angiography is required to look for all sorts of bony, visceral and soft tissue injury including blood vessels.

Patients who are unstable and undergoing CT scan, possibility of cardiac arrest is not uncommon during the scan. Number of specific imaging findings have been observed by different authors which could tell about the imminent nature of cardiac arrest when the patient is on CT couch. These findings are pooling of intravenous contrast in the inferior vena cava and hepatic veins. Hepatic parenchymal contrast reflux and opacification of portal vein. Other findings that could be observed are contrast reflux into the renal veins and venointerstitial reflux. In this case report we have described all these findings alongwith other findings in a patient who was in imminent cardiac arrest during the time of CT examination.

Authors have described strikingly dense contrast opacification of the abdominal vein like inferior vena cava hepatic veins and variable degrees of arterial enhancement and poor abdominal visceral enhancement ${ }^{1,2}$.

Singh et al described opacification of hepatic and renal veins with hepatic parenchymal contrast enhancement. He also described contrast opacification of portal vein ${ }^{3}$.

In a case series published by Jana, they have also mentioned similar findings in their patients. Two of their patients had cardiac arrest during the scan like in our patient and in other two patients, patient went into cardiac arrest soon after the study was done ${ }^{4}$.

Findings of complete cardiac arrest have been described, where there was no opacification of the left sided heart 
chambers and contrast was limited to right atrium with reflux into systemic veins ${ }^{5}$.

Basic pathophysiology of these imaging findings in imminent cardiac arrest is mainly due to sudden and extreme drop in the systemic blood pressure which even falls below the pressure injection of contrast medium through pressure injector during the CT examination. Generally contrast medium is denser than blood but because of the cardiac activity there is thorough mixing of blood with contrast within the cardiac chambers. But during cardiac arrest there is no intermixing with resultant layering of the contrast in the dependant parts of the venous system ${ }^{6}$.

\section{CONCLUSION}

Cardiac arrest during CT examination is of rare occurrence but not so uncommon these days because of the widespread use of $\mathrm{CT}$ in evaluating unstable patients especially in polytrauma cases where cardiac arrest is a possibility due to hemorrhagic shock. The imaging findings are characterstic with pooling of contrast into right sided heart chambers, IVC, hepatic and renal veins and sometimes parenchymal blush due to retrograde reflux. Radiologists and technicians should be aware of these findings so that adequate and timely resuscitative measures can be taken to revive the patient while he is still in CT room.

\section{REFERENCES}

1. Ko SF, Ng SH, Chen MC, Lee TY, Huang CC, Wan YL. Sudden cardiac arrest during computed tomography. J Comput Assist Tomogr. 2003;27(1):93-7.

2. Roth C, Sneider M, Bogot N, Todd M, Cronin P. Dependent venous contrast pooling and layering: A sign of imminent cardiogenic shock. AJR Am J Roentgenol. 2006;186(3):1116-9.

3. Singh AK, Gervais D, Mueller P, Shirkhoda A, Sagar P, Mccarroll K. Cardiac arrest: Abdominal CT imaging features. Abdom Imaging. 2004;29(5):177-9.

4. Jana M, Gamanagatti SR, Kumar A. Case series: $\mathrm{CT}$ scan in cardiac arrest and imminent cardiogenic shock. The Indian journal of radiology and imaging. 2010;20(2):150-153.

5. Shiotani S, Kohno M, Ohashi N, Yamazaki K, Itai Y. Postmortem intravascular high-density fluid level (hypostasis): CT findings. J Comput Assist Tomogr. 2002;26(2):892-3.

6. Tsai PP, Chen JH, Huang JL, Shen WC. Dependent pooling: A contrast-enhanced sign of cardiac arrest during CT. AJR Am J Roentgenol. 2002;178(4):1095-9.

Source of Support: Nil; Conflict of Interest: None

Submitted: 25-04-2018; Accepted: 29-05-2018; Published online: 05-06-2018 